\title{
Competition between ON and OFF Neural Pathways Enhancing Collision Selectivity
}

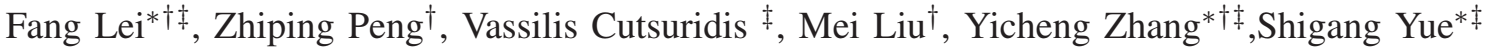 \\ *Joint Research Lab, GDUPT and UoL \\ ${ }^{\dagger}$ Guangdong University of Petrochemical Technology (GDUPT), Maoming, China \\ ${ }^{\ddagger}$ School of Computer Science, University of Lincoln (UoL), Lincoln, UK \\ \{flei, syue,VCutsuridis\}@lincoln.ac.uk, \{pengzp, liumei, zhangyicheng\}@gdupt.edu.cn,
}

\begin{abstract}
The LGMD1 neuron of locusts shows strong looming-sensitive property for both light and dark objects. Although a few LGMD1 models have been proposed, they are not reliable to inhibit the translating motion under certain conditions compare to the biological LGMD1 in the locust. To address this issue, we propose a bio-plausible model to enhance the collisionselectivity by inhibiting the translating motion. The proposed model contains three parts, the retina to lamina layer for receiving luminance change signals, the lamina to medulla layer for extracting motion cues via $\mathrm{ON}$ and $\mathrm{OFF}$ pathways separately, the medulla to lobula layer for eliminating translational excitation with neural competition. We tested the model by synthetic stimuli and real physical stimuli. The experimental results demonstrate that the proposed LGMD1 model has a strong preference for objects in direct collision course-it can detect looming objects in different conditions while completely ignoring translating objects.
\end{abstract}

Index Terms-LGMD1 neuron, neural competition, $\mathrm{ON}$ and OFF pathways, translating motion, inhibition

\section{INTRODUCTION}

Detecting approaching objects from dynamic visual environments is crucial for animals, like locusts, eliciting escape or collision-avoidance behaviors [1]-[3]. The LGMD1 (used to name LGMD) neuron in the locust's visual system, excited by the approach of light or dark objects, plays an important role in such escape or avoidance behaviors [4]. However, how the presynaptic neural networks to implement and the neural computations to shape their responses remain poorly understood [5].

A lot of artificial visual systems based on LGMD1 are still struggling to mimic its collision-selective property [6]-[9]. The bio-plausible structure and computation strategy proposed based on LGMD1 aim to respond to approaching and not translating visual stimuli, as only the former should reliably elicit collision-avoidance behaviors [10]. However, detecting looming as opposed to translating objects is still challenging for existing models due to translational movements may also evoke spike response under certain circumstances.

In [2], the first four-layered LGMD1 neuron model demonstrated that its response would increase with edge velocity when translatory motion across the "eye" at different speeds. It cannot distinguish between the approaching cars and those

This research is funded by the EU HORIZON 2020 project, STEP2DYNA (grant agreement NO. 691154) and ULTRACEPT (grant agreement NO. 778062). nearby fast translating objects in its visual field [11]. Although the fusion of LGMD1 and translating sensitive neural network provide a solution to detect colliding objects in complex dynamic scenes [12], it does not reflect the intrinsic collisionselective property of LGMD1 neuron.

Recently, inspired by biological systems, most models tend to split the input into separate ON and OFF channels for motion detection [13]-[16], which consider the contrast polarity of moving edges [17]. Such dual-pathways processing strategy is also used in collision detection, which combines spike frequency adaptation (SFA) mechanism [18] in shaping the collision selectivity of LGMD1 [7]. The model adopts a method of neural response with derivative profiles to model the biophysical SFA mechanism for enhancing the looming selectivity. However, its computation of membrane potential strongly relies on the tuning of the two adaptation coefficients within a wide range.

For the ON/OFF separated models, there is a question to answer, that is, are the responses from the ON/OFF channels to a looming object and a translating object quite different or similar? We think the responses to looming and translating motion should be quite different within the $\mathrm{ON}$ and $\mathrm{OFF}$ channels separately. This is because a similar amount of paired ON-OFF responses are usually triggered by translating objects but not looming objects. In biology, the neural competition between ON and OFF channels can explain response features arising from opponent inputs [19]. Also, the neurons compete with each other make the winner ones tuned to a certain pattern of inputs but keep the others from becoming selective to that same pattern [20]. Therefore, it is possible to propose a new LGMD1 model by comparing ON and OFF responses.

This paper proposes a new bio-plausible LGMD1 model based on the neural competition between ON and OFF pathways. The morphological LGMD1 neural network and proposed model are as shown in Fig. 1. As can be seen from Fig. 1 , the model separates the ON and OFF channels. The main contribution of this work is comparing $\mathrm{ON}$ and OFF responses for inhibiting translational motion, which effectively enhances the collision selectivity. The rest of this paper is organized as follows: the network architecture of the proposed LGMD1 neural network system is presented in Section 2; explicit experimental results and analysis are provided in Section 3; the discussion is presented in Section 4; finally, we conclude 
this paper in Section 5.

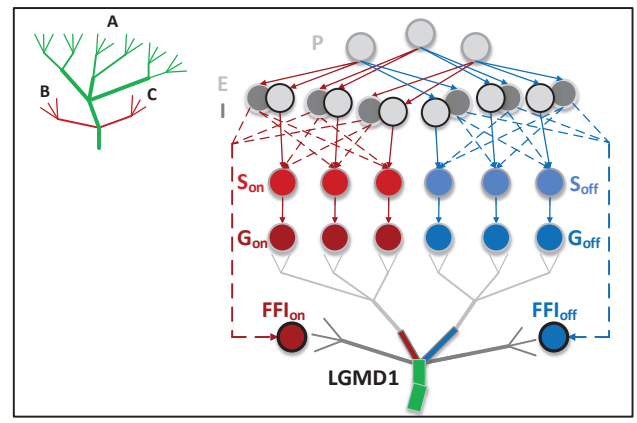

Fig. 1. Schematic illustration of the morphological LGMD1 neural network and proposed model: a large green dendritic field for receiving excitatory synaptic inputs (A), two separate red dendritic fields (B and C) representing $\mathrm{ON}$ and $\mathrm{OFF}$ feed forward inhibition; the model is composed of five layers $(\mathrm{P}, \mathrm{E}, \mathrm{I}, \mathrm{S}, \mathrm{G})$ and three cells $\left(\mathrm{FFI}_{\mathrm{on}}, \mathrm{FFI}_{\mathrm{off}}, \mathrm{LGMD1}\right)$; signals are split into ON (red-arrows) and OFF (blue-arrows) pathways each with four layers (E, I, S, G); excitatory signals make a neural competition in LGMD1 neuron; the dashed lines indicate transmissions of delayed neural signals.

\section{NETWORK ARCHITECTURE}

In this section, we first schematically illustrate the signal processing in LGMD1 model, then elaborate on its components in the following subsections.

The architecture of the proposed neural network is consisted of four layers, including retina, lamina, medulla and lobula layers, as illustrated in Fig.2. The luminance changes are separated into ON and OFF visual stimuli, which represents moving edges of positive or negative contrast polarity. The two opposite stimuli are then further processed in $\mathrm{ON}$ and OFF visual channels and more details are as follows.

\section{A. Retina to Lamina layer}

The luminance (L) signals are received by photoreceptors and converted into electrical signals. The output of $\mathrm{P}$ cells is defined by equation:

$$
P_{t}(x, y)=L_{t}(x, y)-L_{t-i}(x, y)
$$

where $P_{t}(x, y)$ reflects the luminance change of pixel $(x, y)$ over time $i . L_{t}(x, y)$ and $L_{t-i}(x, y)$ indicate the gray value of pixel $(x, y)$ at time $t$ and $t-i$. For video image processing, the $\mathrm{P}$ cells are arranged in a matrix and continuous time is discretized by frames. Time $t$ and $t-i$ represent two successive frames with the frame interval $i$ decided by frame rate. Different frame rate affects the outputs of $\mathrm{P}$ cells due to moving edges of various extent.

\section{B. Lamina to Medulla layer}

As shown in Fig. 2, the intensity increments and decrements are rectified by transient cells in the medulla [21]. The two types of stimuli are further processed with three processes in $\mathrm{ON}$ and OFF pathways separately. It is noted that ON and OFF pathways show the computational process similarly, which is elaborated as follows.

1) Asymmetric Mechanism: The $P$ cell corresponds to a pairwise excitatory (E) and inhibitory (I) cell. Its polarity of positive (ON) value do not change but the negative (OFF) is converted into positive, which corresponds to half-wave rectification' [22]:

$$
\begin{gathered}
E_{t}^{O N}(x, y)=I_{t}^{O N}(x, y)=\left[P_{t}(x, y)\right]^{+} \\
E_{t}^{O F F}(x, y)=I_{t}^{O F F}(x, y)=-\left[P_{t}(x, y)\right]^{-}
\end{gathered}
$$

where $[a]^{+}=\max (0, a),[a]^{-}=\min (0, a), \mathrm{E}$ and I units in both $\mathrm{ON}$ and OFF channels have the same value related to the the image contrast.

2) Lateral Inhibition Mechanism: The lateral inhibition mechanism is a common feature of early visual processing in many organisms [23]. In our model, lateral inhibition process is described: center excitation unit (CEU) and output of time delay unit (TDU) passed from surround inhibition unit (SIU) are summed by $\mathrm{S}$ unit (see Fig. 2).

$$
\begin{aligned}
S_{-} I_{t}^{O N}(x, y) & =\sum_{i=-1}^{1} \sum_{j=-1}^{1} I_{t-\tau_{s}}^{O N}(x+i, y+j) W_{i}(i, j) \\
S_{t}^{O N}(x, y) & =\left[E_{t}^{O N}(x, y)-S_{-} I_{t}^{O N}(x, y)\right]^{+}
\end{aligned}
$$

where $S_{-} I_{t}^{O N}(x, y)$ represents the lateral summed inhibitory signals correspond to spatial position $(x, y)$ at time $\mathrm{t}$ in $\mathrm{ON}$ pathway, $\tau_{s}$ represents the time delay constant, $W_{i}$ is the local inhibition connection weight matrix (see Fig. 3 (a), (b)). $S_{t}^{O N}(x, y)$ denotes the output of S cell.

3) Group-decay Processing Mechanism: The group-decay process [1] is efficient and reliable for eliminating small and isolated excitations in background. In addition, the grouping operation connects lateral excitations together that enhances the response to coherent stimuli [5]. It can be mathematically defined as:

$$
\begin{gathered}
C e_{t}^{O N}(x, y)=\sum_{i=-1}^{1} \sum_{j=-1}^{1} S_{t}^{O N}(x+i, y+j) W_{s}(i, j) \\
\left.\omega=\triangle c+\max \left(a b s[C e]_{t}^{O N}\right)\right) C_{w}^{-1} \\
G_{t}{ }^{O N}(x, y)=\left(S_{t}^{O N}(x, y)\right) C e_{t}^{O N}(x, y) \omega^{-1} \\
\tilde{G}_{t}^{O N}(x, y)= \begin{cases}G_{t}^{O N}(x, y) C_{d e} & \text { if } G_{t}^{O N}(x, y) \geq T_{g} \\
0 & \text { else }\end{cases}
\end{gathered}
$$

where $C e_{t}^{O N}$ denotes the passing coefficient (see (5a)). $\omega$ is a scale that is computed by (5b), where $\triangle c$ is a small real number and $C_{w}$ is a constant.

The $\mathrm{S}$ unit matrix is firstly convolved with a weighting matrix $\left(W_{s}\right)$ of group connection to compute the passing coefficient. Then, the $\mathrm{S}$ unit is multiplied by this passing coefficient, which connects directly to the G unit (see Fig. 3 (a), (c)). The decayed excitation remain unexcited as denoted in (5c) and (5d), where $C_{d e}$ and $T_{g}$ indicate the decay coefficient and threshold, $C_{d e} \in(0,1)$. When the grouped excitation $G_{t}{ }^{O N}$ exceeds the decay threshold, its value $\tilde{G}_{t}^{O N}$ will pass from the G cell to LGMD1 neuron. 


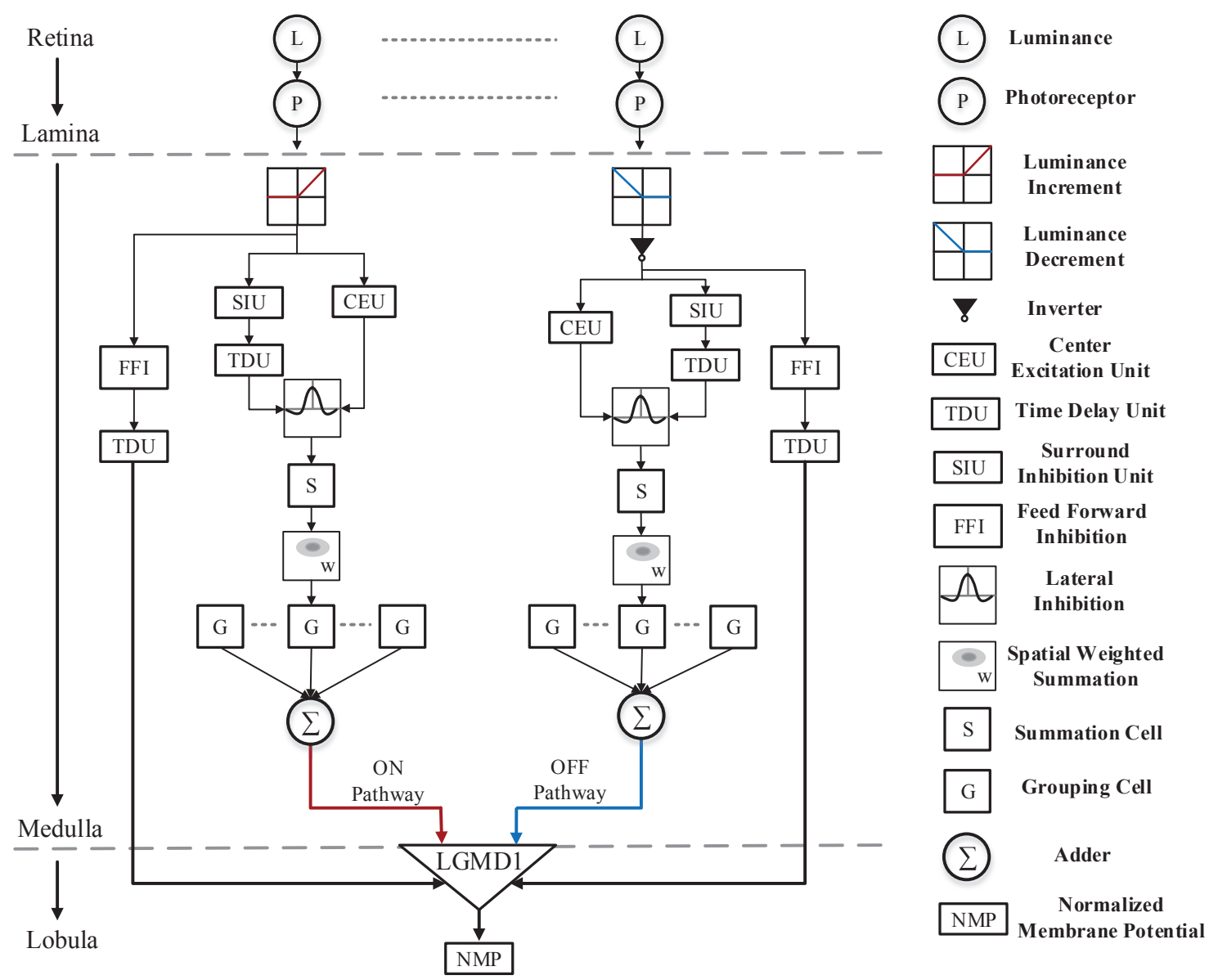

Fig. 2. Schematic illustration of signal processing in LGMD1 model, the procedure is divided into three processing stages: retina to lamina layer, the photoreceptor $(\mathrm{P})$ cells capture pixel-wise luminance (L) change; lamina to medulla layer, opposite visual stimuli are processed separately in ON and OFF pathways; medulla to lobula layer, the winner excitatory signals are converged into LGMD1 neuron after a neural competition between ON and OFF pathways; the combined delayed feed forward inhibition (FFI) signals inhibit the model's initial response to movement.

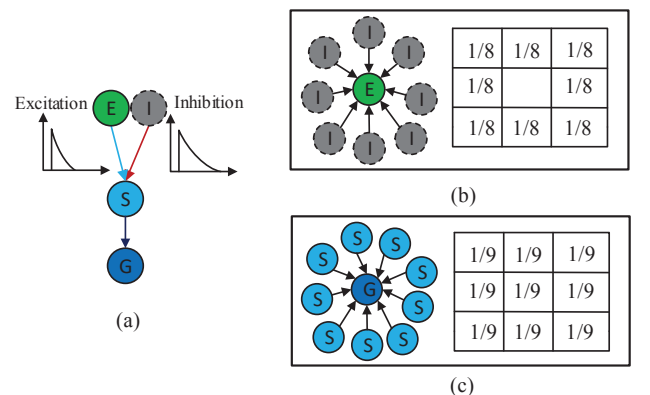

Fig. 3. Schematic illustration of lateral inhibition and group-decay processing mechanism in lamina to medulla layer: (a) the E unit passes excitation to $S$ unit in the same retinotopic position and each surrounding delayed I unit passes inhibition to $\mathrm{S}$ unit simultaneously where excitation and inhibition decay exponentially with different coefficients (delays at excitatory connections are set to 0 milliseconds, whereas delays on inhibitory connections are set to vary from several to tens of milliseconds accordingly); (b) weighting matrix of lateral inhibition connection; (c) weighting coefficients matrix of group connection.

\section{Medulla to Lobula layer}

Many trans-medullary-afferents (TmAs) connect the eye with each LGMD in the lobula layer [24]. The transmedullary afferents of ON and OFF pathways pass excitatory signals from $\mathrm{G}$ cells to LGMD1. A neural competition between signals will elicit a spike if the membrane potential exceeds a fixed threshold. The neural competition process schematically illustrated in Fig. 4, but the exact mapping of cells in the model is not known.

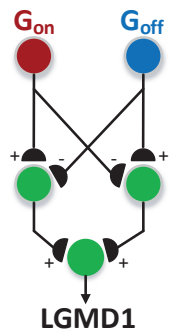

Fig. 4. Schematic illustration of the neural competition process indicating responses comparison between ON and OFF channels. $G_{O N}$ and $G_{O F F}$ are effective outputs (nonzero value) of G layer participating in competition. The winner excitations are passed to LGMD1 neuron.

Neural Competition Mechanism: To implement the neural competition mechanism, the nonzero outputs of G cells in ON 
and OFF channels are summed separately. Then the winner excitation are summed and passed to the LGMD1 neuron. The process can be mathematically described as:

$$
\begin{aligned}
C_{t}^{O N} & =\sum_{x} \sum_{y} \tilde{G}_{t}^{O N}(x, y) \\
C_{t}^{O F F} & =\sum_{x} \sum_{y} \tilde{G}_{t}^{O F F}(x, y)
\end{aligned}
$$

where $C_{t}^{O N}$ and $C_{t}^{O F F}$ denote nonzero excitation in two opponent pathways, which also represents the practical expanding stimuli. The membrane potential $\mathrm{MP}(\mathrm{t})$ value is defined as:

$$
\begin{gathered}
C_{\max }=\max \left(C_{t}^{O N}, C_{t}^{O F F}\right) \\
C_{\min }=\min \left(C_{t}^{O N}, C_{t}^{O F F}\right) \\
\mathrm{MP}(\mathrm{t})= \begin{cases}C_{\max }-C_{\min } & \text { if } C_{\min } \leq 1 \\
C_{\max } / C_{\min }-1 & \text { else }\end{cases}
\end{gathered}
$$

where the value of $\mathrm{MP}(\mathrm{t})$ is scaled that characterize the saturation of neuronal response to large input. It is then transformed to normalized membrane potential $\mathrm{NMP}(t)$ as

$$
\operatorname{NMP}(t)=1-\left(1 / \exp \left(\operatorname{MP}(\mathrm{t}) \cdot \mathrm{n}_{\text {cell }}^{-1}\right)\right)
$$

where $n_{\text {cell }}$ is the total number of the cells in $\mathrm{G}$ layer, $\operatorname{NMP}(t) \in(0 \sim 1)$.

\section{Spiking mechanism}

The spiking mechanism will decide whether the model trigger a collision alarm. If the normalized membrane potential NMP $(t)$ exceeds the threshold $T_{s}$, it produces a spike,

$$
\text { Spike }(t)= \begin{cases}1 & \text { if } \operatorname{NMP}(t) \geq T_{s} \\ 0 & \text { otherwise }\end{cases}
$$

where 1 represents a spike, 0 means no spike. A collision alarm is detected when there are successive $n_{s p}$ spikes in time $t_{n}$ [1]. That is,

$$
C_{\text {alarm }}(t)= \begin{cases}T R U E & \text { if } \sum_{i=t-t_{n}}^{t} \text { Spike }(i) \geq n_{s p} \\ F A L S E & \text { otherwise }\end{cases}
$$

where the value of $C_{\text {alarm }}(t)$ will become TRUE if a collision is detected. The successive number of spikes $n_{s p}$ is usually set four.

\section{E. The Feed Forward Inhibition (FFI)}

In the ON and OFF visual pathways, the FFI signals are gathered from the $\mathrm{P}$ cells with tens of milliseconds delay $(\tau)$. The effect of feed forward inhibition is to suppress the model's initial response to movement [23]. Once the value of FFI exceeds its threshold, spikes in the LGMD are inhibited immediately. It can be described by the following function.

$$
F F I=\sum_{x=1}^{n_{r}} \sum_{y=1}^{n_{c}} P_{t-\tau}^{O N}(x, y)+\sum_{x=1}^{n_{r}} \sum_{y=1}^{n_{c}} P_{t-\tau}^{O F F}(x, y)
$$

\section{EXPERIMENTS AND ANALYSIS}

In this section, we will present the systematic experiments to evaluate the performance of our proposed model. It consists two parts: test the competition hypothesis by analyzing the polarity properties of moving edges and present the different response properties between $\mathrm{ON}$ and OFF visual pathways by synthetic stimuli; verify the efficiency of the proposed LGMD1 model by real-world physical stimuli. The parameters of the neural network in all experiments were kept the same.

\section{A. Analysis and Results on Synthetic Stimuli}

We analysed the approaching and translating motion in a simulated environment (see Fig. 5 ) to obtain a deep insight into the computational model. It shows various response properties on luminance changes comparing with two types of motion in an image plane. To further demonstrate the previous

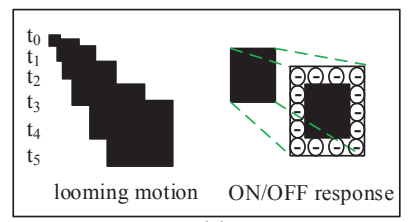

(a)

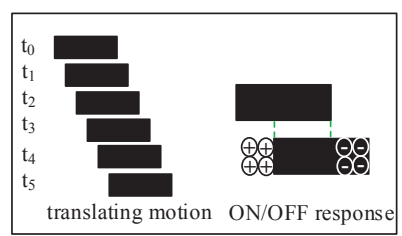

(c)

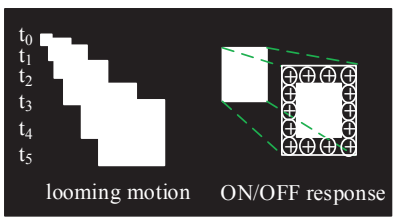

(b)

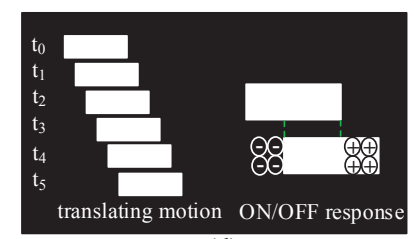

(d)

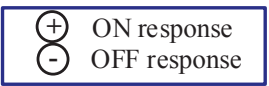

Fig. 5. Schematic illustration of stimuli properties between looming and translating motion: (a) and (b) represent a black and white square looming in a white and black background separately; (c) and (d) represent a black and white rectangle translating in a white and black background separately. The looming motion shows the monotonous response while translating motion shows the pair of ON/OFF response.

hypothesis in the proposed neural network, we observe and analyze the outputs of $\mathrm{ON}$ and OFF channels by testing synthetic stimuli of looming and translating motion. The resolution of the synthesized image sequences is $300 \times 300$.

Fig. 6(a) and (b) simulate the looming motion of the dark and light object. As can be seen, the excitation response linearly increases in a single pathway of OFF or ON as the expanding of moving edges. This indicates that the responses in $\mathrm{ON}$ and $\mathrm{OFF}$ pathways are quite different for looming motion.

Fig. 7(a), (b) and Fig. 8(a), (b) simulate the translating motion of the dark and light object in a different direction, which indicates that the excitation response in both $\mathrm{ON}$ and OFF pathways are very similar in spite of the motion's direction. 


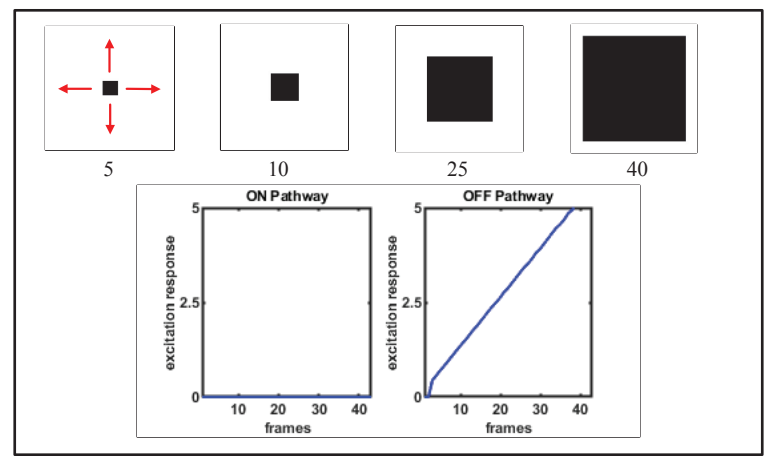

(a)

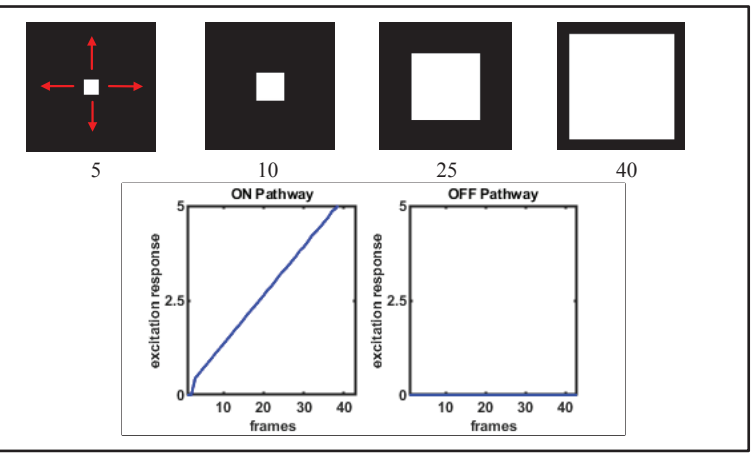

(b)

Fig. 6. The excitation response of looming objects in ON and OFF pathways: (a) there are 43 frames featuring a dark square in a light background expanding linearly; (b) there are 43 frames featuring a light square in a dark background expanding linearly.

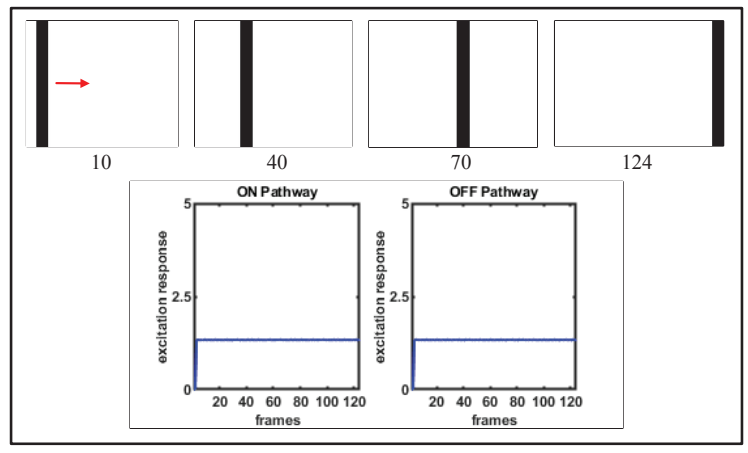

(a)
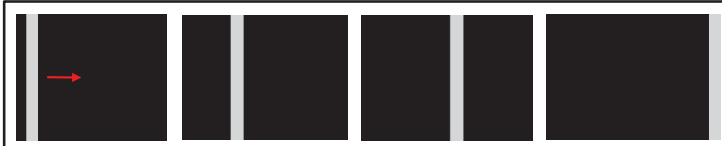

40 124

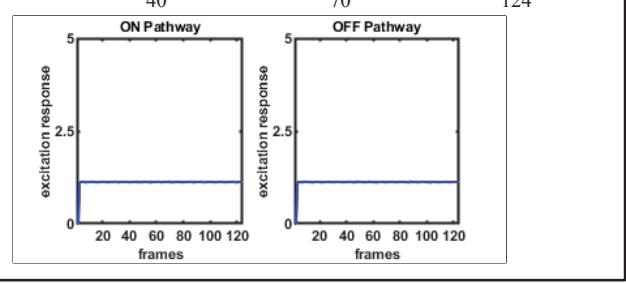

(b)

Fig. 7. The excitation response of translating objects in $\mathrm{ON}$ and OFF pathways: (a) there are 124 frames featuring a dark bar in a light background move from left to right; (b) there are 124 frames featuring a light bar in a dark background move from left to right.

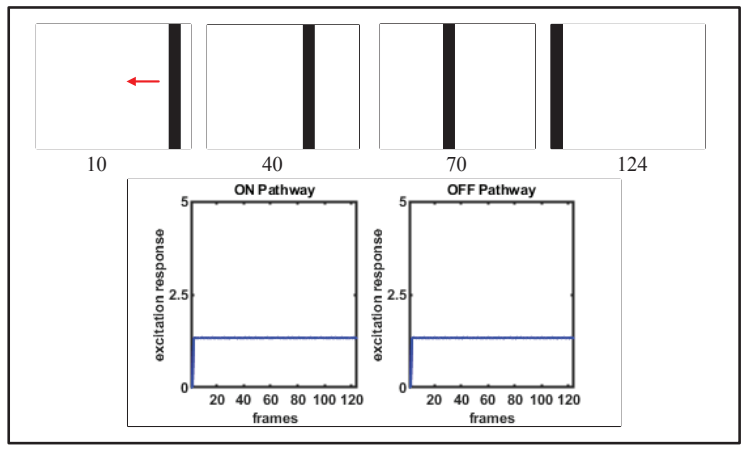

(a)

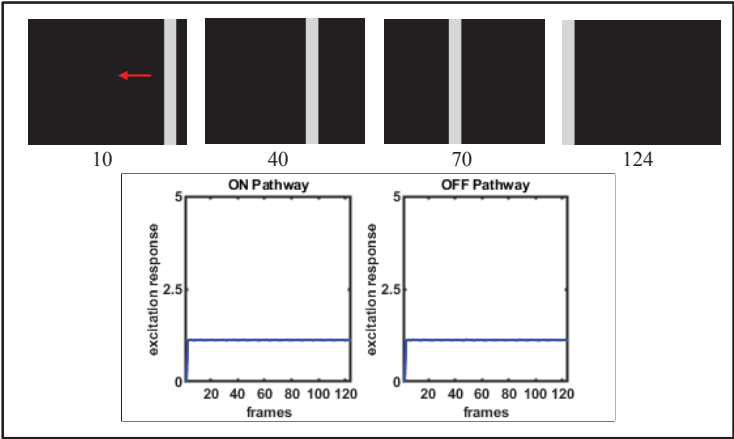

(b)

Fig. 8. The excitation response of translating objects in $\mathrm{ON}$ and $\mathrm{OFF}$ pathways: (a) there are 124 frames featuring a dark bar in a light background move from right to left; (b) there are 124 frames featuring a light bar in a dark background move from right to left.

\section{B. Challenged by Real Stimuli}

To test whether the proposed model works reliably, the best way is to challenge it with real-world physical stimuli. We tested the model with eight recorded video clips. The former six image sequences provide looming/approaching and translating movements under various conditions. We also consider translatory movements in the visual field when the camera is turning, which are shown at the last two image sequences. These image sequences have resolutions of $100 \times 120$, $100 \times 125$ and $100 \times 150$ at 30 frames per second (fps).

Fig. 9, Fig. 10 and Fig. 11 show the collision trajectory including the looming object or the camera approaching block respectively. The colliding objects have different colors, sizes, and shapes. The experimental results demonstrated that the proposed LGMD1 neuron model can successfully detect the impending collision. Comparing with three response curves, they also indicate that the faster-speed and higher-contrast visual stimuli will make the model produce spikes earlier than the opposite.

Fig. 12, Fig. 13 and Fig. 14 show objects translating in front of the camera from left to right or from right to left. The experimental results demonstrated that the model can strongly inhibit the translational motion despite objects' own various colors, sizes, and shapes. The unexpected three spikes in Fig. 14 are caused by the near-white ball's uneven surface reflection or shadows. Note that the near-fast or high contrast objects abruptly move in or out of the field of view may also trigger 

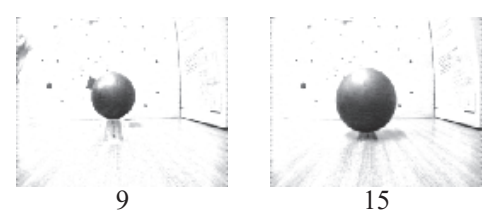

15
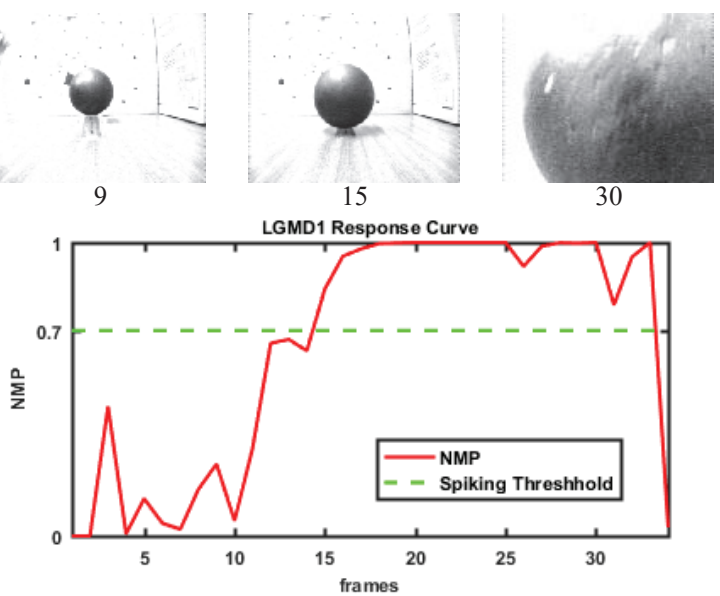

Fig. 9. The neural response of the proposed model challenged by physical stimuli from the first recorded image sequence. There are 34 frames featuring a black ball looming to the camera.
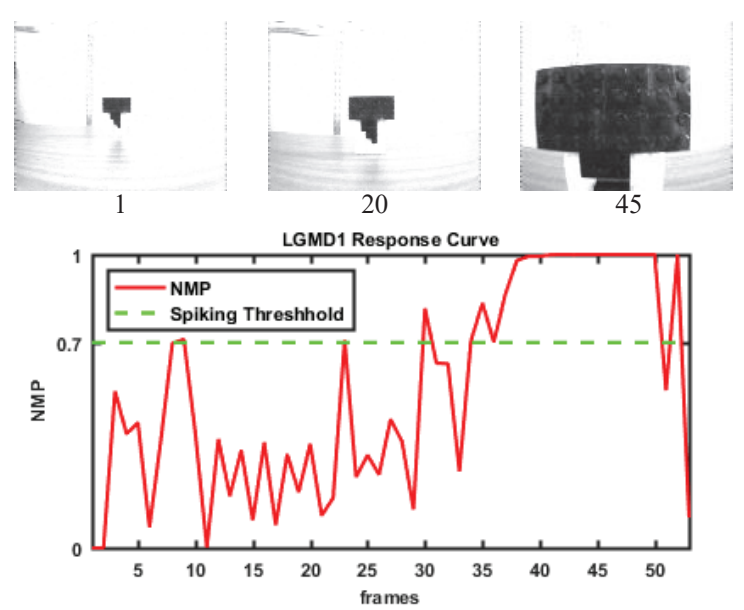

Fig. 10. The neural response of the proposed model challenged by physical stimuli from the second recorded image sequence. There are 53 frames featuring the camera approaching a gray block.
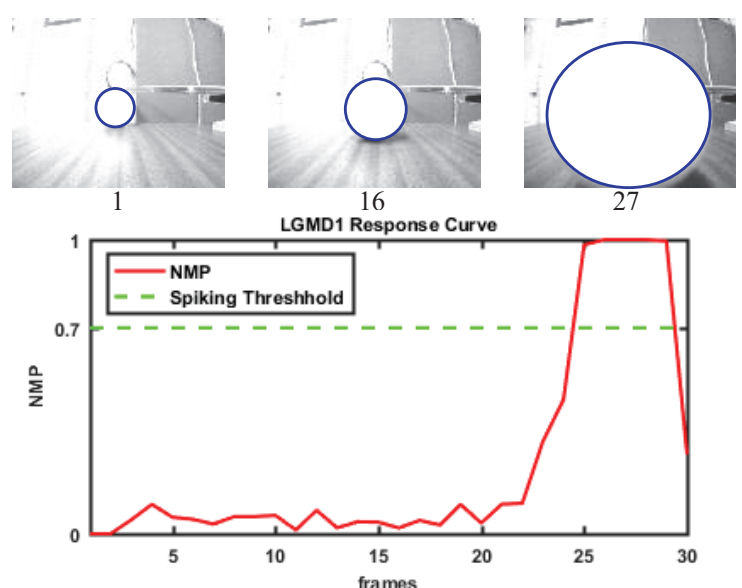

Fig. 11. The neural response of the proposed model challenged by physical stimuli from the third recorded image sequence. There are 30 frames featuring a white ball looming to the camera and it was marked by a blue circle in the image.

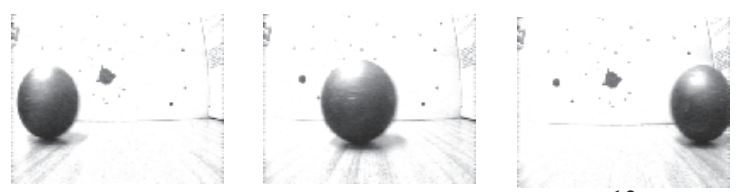

1

6

13

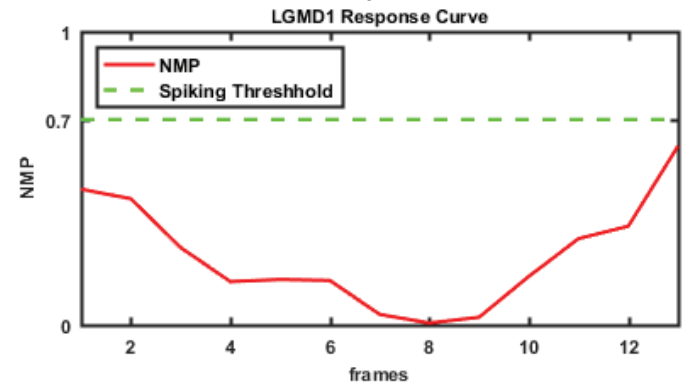

Fig. 12. The neural response of the proposed model challenged by physical stimuli from the fourth recorded image sequence. There are 13 frames featuring a black ball translating in front of the camera from left to right.

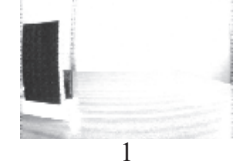

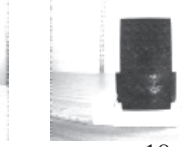

18

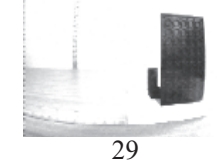

29

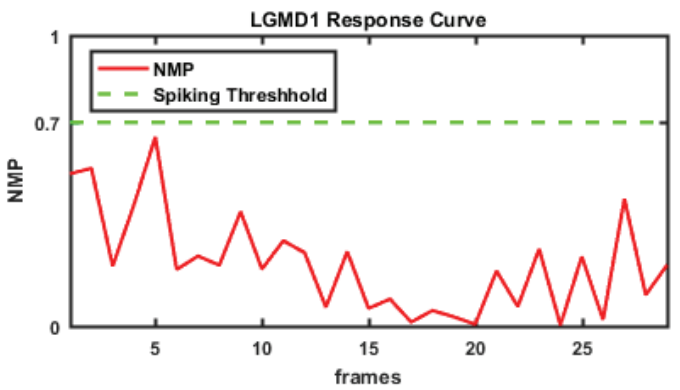

Fig. 13. The neural response of the proposed model challenged by physical stimuli from the fifth recorded image sequence. There are 29 frames featuring a gray block translating in front of the camera from left to right.
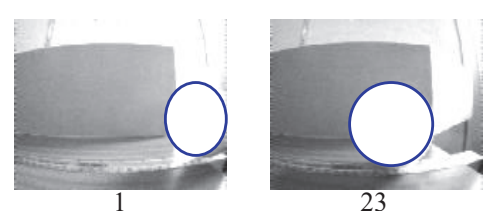

23
LGMD1 Response Curve
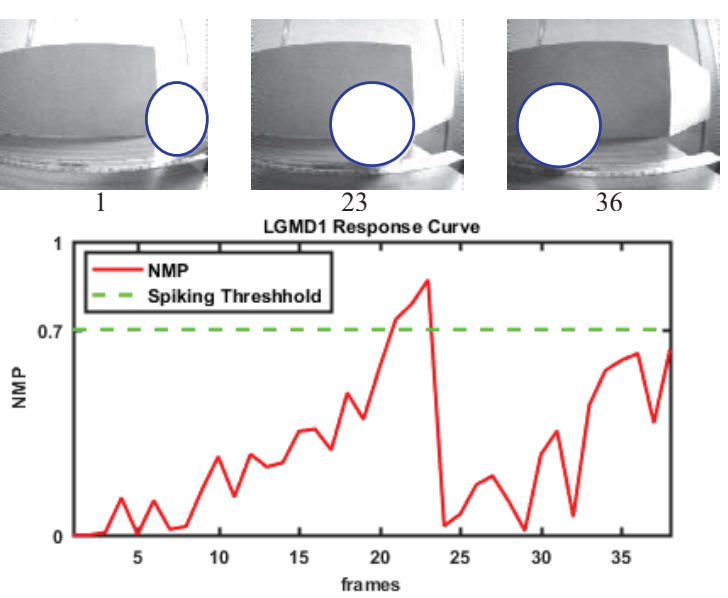

Fig. 14. The neural response of the proposed model challenged by physical stimuli from the sixth recorded image sequence. There are 38 frames featuring a white ball translating in front of the camera from right to left. The white ball was marked by a blue circle in the image. 
the model's spiking. This phenomenon can be regarded as events occur or it can be eliminated by smoothing the output data, which depends on the need of the practical situation.

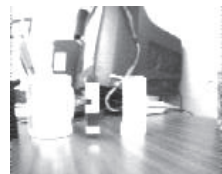

10

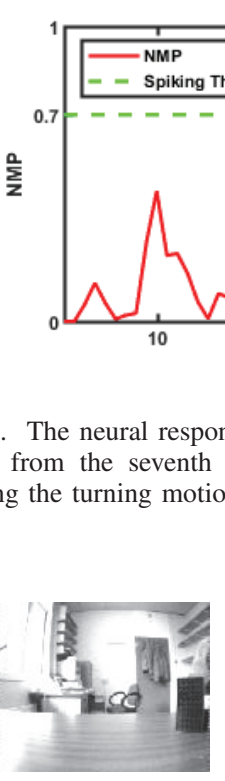

10

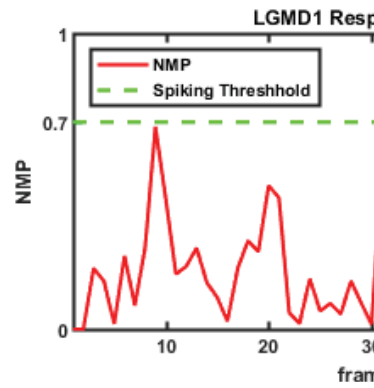

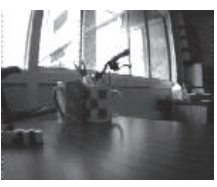

ponse Curve

rames

50
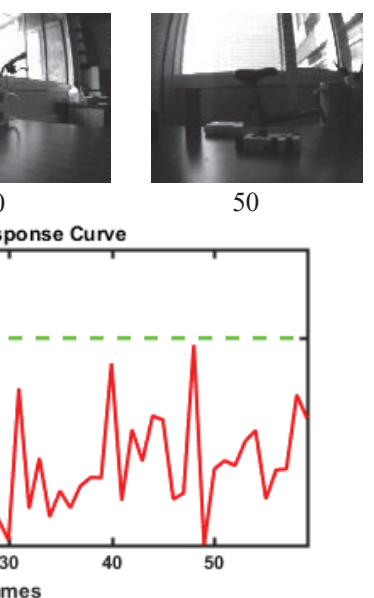

Fig. 16. The neural response of the proposed model challenged by physical stimuli from the eighth recorded image sequence. There are 59 frames featuring the turning motion of the camera under non-uniform illumination.

Fig. 15 and Fig. 16 show the turning motion of the camera under different lighting conditions. The experimental results demonstrate that translational movements caused by the turning motion of the camera can also be successfully inhibited.

\section{Discussion}

In the above section, we have proposed a new LGMD1 model using competition between $\mathrm{ON}$ and OFF neural pathways enhancing collision selectivity. The experiments showed that it worked well especially when challenged with translating motion cues. In the lobula of the locust optic lobe, there are many other neurons working seamlessly together to respond to the dynamic visual stimuli [25]. For example, there are LGMD2 [4], [26]-[28] which only respond to darker objects and directional selective neurons which only excited by visual motion to specific direction [29], [30]. It is worth combing all these neurons' functionalities together in the future work to provide robust solutions for collision detection for robotics and autonomous vehicles.

\section{CONCLUSION AND FUTURE WORK}

To summarize, we have proposed a bio-plausible LGMD1 model for enhancing the collision selectivity. This new model based on the neural competition can tell looming and translating motion by comparing $\mathrm{ON}$ and $\mathrm{OFF}$ responses. Both the synthetic stimuli tests and real-world physical stimuli experiments have demonstrated that the neural competition between the opponent visual pathways is effective for inhibiting translatory objects and sensitive to looming objects. It should be noted that the suddenly appeared or disappeared object in the visual field may trigger the model spiking. However, the above-mentioned situation may not happen for insects since they own a nearly 360-degree field of view.

In our future work, we will investigate the potential applications of neural competition mechanism integrated into the LGMD1 model to handle more complex and dynamic visual scenes for the navigation of robots and vehicles.

\section{REFERENCES}

[1] S. Yue and F. C. Rind, "Collision detection in complex dynamic scenes using an lgmd-based visual neural network with feature enhancement," IEEE Trans. Neural Netw., vol. 17, no. 3, pp. 705-716, 2006.

[2] F. C. Rind and D. Bramwell, "Neural network based on the input organization of an identified neuron signaling impending collision," $J$. Neurophysiol., vol. 75, no. 3, pp. 967-985, 1996.

[3] F. Gabbiani, H. G. Krapp, and G. Laurent, "Computation of object approach by a wide-field, motion-sensitive neuron," J. Neurosci., vol. 19, no. 3, pp. 1122-1141, 1999.

[4] P. J. Simmons and F. C. Rind, "Responses to object approach by a wide field visual neurone, the lgmd 2 of the locust: Characterization and image cues," J. Comp. Physiol. - A Sensory, Neural, Behav. Physiol., vol. 180, no. 3, pp. 203-214, 1997.

[5] Y. Zhu, R. B. Dewell, H. Wang, and F. Gabbiani, "Pre-synaptic muscarinic excitation enhances the discrimination of looming stimuli in a collision-detection neuron," Cell reports, vol. 23, no. 8, pp. 2365-2378, 2018.

[6] C. Hu, F. Arvin, C. Xiong, and S. Yue, "Bio-inspired embedded vision system for autonomous micro-robots: the lgmd case," vol. 9, no. 3, pp. 241-254, 2016.

[7] Q. Fu, C. Hu, J. Peng, and S. Yue, "Shaping the collision selectivity in a looming sensitive neuron model with parallel on and off pathways and spike frequency adaptation," Neural Networks, vol. 106, pp. 127-143, 2018.

[8] B. Hu, Z. Zhang, and L. Li, "Lgmd-based visual neural network for detecting crowd escape behavior," in 2018 5th IEEE International Conference on Cloud Computing and Intelligence Systems (CCIS). IEEE, 2018, pp. 772-778.

[9] J. Zhao, X. Ma, Q. Fu, C. Hu, and S. Yue, "An lgmd based competitive collision avoidance strategy for uav," in IFIP International Conference on Artificial Intelligence Applications and Innovations. Springer, 2019, pp. 80-91.

[10] S. Peron and F. Gabbiani, "Spike frequency adaptation mediates looming stimulus selectivity in a collision-detecting neuron," Nat. Neurosci., vol. 12, no. 3, p. 318, 2009.

[11] S. Yue, F. C. Rind, M. S. Keil, J. Cuadri, and R. Stafford, "A bio-inspired visual collision detection mechanism for cars: Optimisation of a model of a locust neuron to a novel environment," Neurocomputing, vol. 69, no. $13-15$, pp. $1591-1598,2006$.

[12] S. Yue and F. C. Rind, "Visual motion pattern extraction and fusion for collision detection in complex dynamic scenes," Comput. Vis. Image Underst., vol. 104, no. 1, pp. 48-60, 2006. 
[13] K. Shinomiya, S.-y. Takemura, P. K. Rivlin, S. M. Plaza, L. K. Scheffer, and I. A. Meinertzhagen, "A common evolutionary origin for the on-and off-edge motion detection pathways of the drosophila visual system," Front. Neural Circuits, vol. 9, p. 33, 2015.

[14] H. Wang, J. Peng, and S. Yue, "A directionally selective small target motion detecting visual neural network in cluttered backgrounds," IEEE Trans. Cybern., pp. 1-14, 2018.

[15] H. Wang, J. Peng, P. Baxter, C. Zhang, Z. Wang, and S. Yue, "A model for detection of angular velocity of image motion based on the temporal tuning of the drosophila," in International Conference on Artificial Neural Networks. Springer, 2018, pp. 37-46.

[16] H. Wang, J. Peng, X. Zheng, and S. Yue, "A robust visual system for small target motion detection against cluttered moving backgrounds," in press.

[17] M. Joesch, B. Schnell, S. V. Raghu, D. F. Reiff, and A. Borst, "On and off pathways in drosophila motion vision," Nature, vol. 468, no. 7321 , p. 300, 2010.

[18] F. Gabbiani and H. G. Krapp, "Spike-frequency adaptation and intrinsic properties of an identified, looming-sensitive neuron," J. Neurophysiol., vol. 96, no. 6, pp. 2951-2962, 2006.

[19] H. Öĝmen and S. Gagné, "Neural models for sustained and on-off units of insect lamina," Biol. Cybern., vol. 63, no. 1, pp. 51-60, 1990.

[20] D. Krotov and J. J. Hopfield, "Unsupervised learning by competing hidden units," Proceedings of the National Academy of Sciences, vol. 116, no. 16, pp. 7723-7731, 2019.

[21] D. Osorio, "Mechanisms of early visual processing in the medulla of the locust optic lobe: how self-inhibition, spatial-pooling, and signal rectification contribute to the properties of transient cells," Vis. Neurosci. vol. 7, no. 4, pp. 345-355, 1991.

[22] R. Behnia, D. A. Clark, A. G. Carter, T. R. Clandinin, and C. Desplan, "Processing properties of on and off pathways for drosophila motion detection," Nature, vol. 512, no. 7515, pp. 427-430, 2014.

[23] R. D. Santer, R. Stafford, and F. C. Rind, "Retinally-generated saccadic suppression of a locust looming-detector neuron: investigations using a robot locust," J. R. Soc. Interface, vol. 1, no. 1, pp. 61-77, 2004.

[24] F. C. Rind, S. Wernitznig, P. Pölt, A. Zankel, D. Gütl, J. Sztarker, and G. Leitinger, "Two identified looming detectors in the locust: ubiquitous lateral connections among their inputs contribute to selective responses to looming objects," Sci. Rep., vol. 6, no. June, pp. 1-16, 2016.

[25] F. C. Rind, "Non-directional, movement sensitive neurones of the locust optic lobe," J. Comp. Physiol. A, vol. 161, no. 3, pp. 477-494, 1987.

[26] J. Sztarker and F. C. Rind, "A look into the cockpit of the developing locust: Looming detectors and predator avoidance," Dev. Neurobiol., vol. 74, no. 11, pp. 1078-1095, 2014.

[27] Q. Fu, C. Hu, T. Liu, and S. Yue, "Collision selective lgmds neuron models research benefits from a vision-based autonomous micro robot," in 2017 IEEE/RSJ International Conference on Intelligent Robots and Systems (IROS). IEEE, 2017, pp. 3996-4002.

[28] Q. Fu, C. Hu, J. Peng, F. C. Rind, and S. Yue, "A robust collision perception visual neural network with specific selectivity to darker objects," IEEE Trans. Cybern., in press.

[29] F. C. Rind, "Identification of directionally selective motion-detecting neurones in the locust lobula and their synaptic connections with an identified descending neurone," J. Exp. Biol., vol. 149, no. 1, pp. 21-43, 1990.

[30] S. Yue and F. C. Rind, "Postsynaptic organizations of directional selective visual neural networks for collision detection," Neurocomputing, vol. 103, pp. 50-62, 2013. 